\title{
Refractive index engineering in glass containing spherical silver nanoparticles using dc electric field
}

\author{
Amin Abdolvand, Alexander Podlipensky, Gerhard Seifert \\ Physics Department, Martin-Luther-University, Hoher Weg, Halle D-06099, Germany \\ e-mail:abdolvand@physik.uni-halle.de \\ Olivier Deparis \\ Electromagnetism \& Telecommunications Department, Faculté Polytechnique de Mons, Mons, Belgium \\ Peter G. Kazansky \\ Optoelectronics Research Centre, University of Southampton, Southampton, United Kingdom \\ Heinrich Graener \\ Physics Department, Martin-Luther-University, Hoher Weg 8, Halle D-06099, Germany
}

Lately composite materials containing metal nanoparticles have increasingly found various applications in different fields of science and technology. In this context glasses containing metallic nanoparticles are of great interest because of their application in the field of photonics owing to their unique linear and nonlinear optical properties. The linear and nonlinear optical properties of such materials are determined by surface plasma oscillations of the metal clusters. The surface plasmon (SP) resonance depends strongly on shape, distribution and concentration of the nanoparticles as well as on the surrounding dielectric matrix. This offers the opportunity to manufacture very promising nonlinear materials, nanodevices and optical elements by manipulation of the nanostructural properties of nanocomposite media.

Recently, we have demonstrated destruction and dissolution of silver nanoparticles embedded in glass matrix by applying a combination of an intense DC electric field and moderately elevated temperature [1,2]. The phenomenon was physically interpreted in terms of ionisation of the metal nanoclusters followed by the removal of ions from the clusters and their drift in the depth of the glass substrate.

Here, using the technique described in Ref. [1], we demonstrate the possibility to structure a buried layer of percolated silver clusters in a glass containing spherical silver nanoparticles. This in principle allows to engineer optical properties of the material via gaining control over spatial distribution of silver in the glass.

Sample used in our experiment was prepared from soda-lime float glass by $\mathrm{Ag}^{+}-\mathrm{Na}^{+}$ion exchange and following annealing in $\mathrm{H}_{2}$ reduction atmosphere. The final product had spherical silver nanoparticles of 30-40nm mean diameter in a thin surface layer of approximately $6 \mu \mathrm{m}$ thickness. The experiment was performed by equipping the sample with two pressed contact electrodes on the surfaces, the anode facing the layer containing nanoparticles. The sample was heated to a temperature of $\sim 250^{\circ} \mathrm{C}$, and then the dc voltage was applied and increased stepwise (up to max. value of $1 \mathrm{kV}$ in steps of $0.2 \mathrm{kV}$ within a total time of $50 \mathrm{~min}$ ). The current was less than $200 \mu \mathrm{A}$ at any time. Finally the voltage was disconnected and the temperature was reduced down to the ambient temperature. This resulted in production of a buried layer of percolated silver clusters beneath the glass surface. A rainbow like pattern was observed in reflection from the sample surface (fig. 1) where the anode was placed, in the border region between the treated area (fig. 1-1) and the original (fig. 1-2), untreated, part of the sample. Observation of the pattern in the border region is attributed to the interference between the light reflected from the sample surface and the light reflected from the engineered buried layer in the border region, where the thickness is nonuniform. The physical mechanism behind the formation of the buried layer as well as the possibility of gaining control over the distance between the buried layer and the surface in order to fulfil the interference condition required to observe large areas with certain colour will be discussed in detail.

Figure 1: Photographs of a segment of anodic surface of the sample after treatment at $1 \mathrm{kV}, 250^{\circ} \mathrm{C}$. Photographs were taken in: Left) reflection and Right) transmission modes of a microscope spectrophotometer [MPM $800 \mathrm{D} / \mathrm{UV}$, Zeiss] equipped with CCD camera. As it can be seen in transmission the pattern has disappeared.
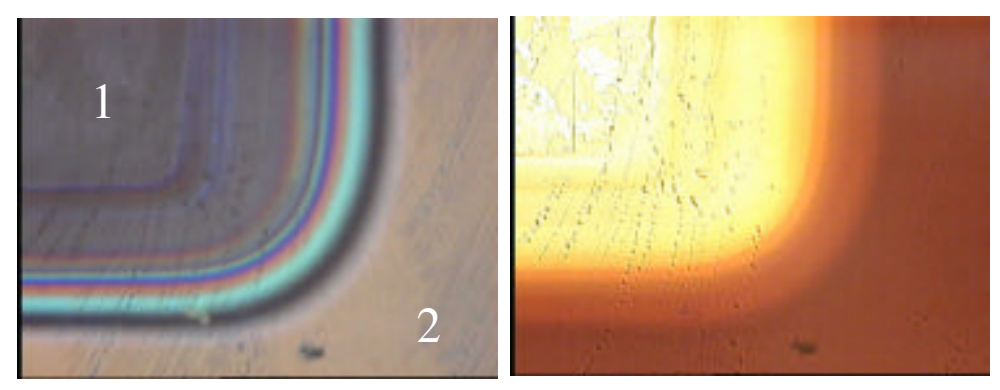

\section{References}

1. A. Podlipensky, A. Abdolvand, G. Seifert, H. Graener, O. Deparis, P. G. Kazansky, J. of Phys. Chem. B 108, 17699 (2004).

2. O. Deparis, P.G. Kazansky, A. Abdolvand, A. Podlipensky, G. Seifert, H. Graener, Appl. Phys, Lett. 85, 872 (2004). 